\title{
Assessment the Possibility of Radiation Dermatitis Incidence in the Interventionist's Leg
}

\author{
Fariba Zarei, ${ }^{1,}{ }^{*}$ Alireza Rasekhi, ${ }^{1}$ Banafsheh Zeinali-Rafsanjani, ${ }^{2}$ Mahdi Saeedi-Moghadam, ${ }^{1}$ and Amin
}

\author{
Abolhasani Foroughi ${ }^{1}$ \\ ${ }^{1}$ Shiraz University of Medical Science, Shiraz, Iran \\ ${ }^{2}$ Medical Imaging Research Center, Shiraz University of Medical Science, Shiraz, Iran \\ "Corresponding author: Fariba Zarei, Shiraz University of Medical Science, Shiraz, Iran. E-mail: zareifari@yahoo.com
}

Received 2016 December 21; Accepted 2017 February 08.

\begin{abstract}
Background: Fluoroscopy guided imaging, which is going to be more routine these days require a long time of fluoroscopic observing, which can increase the radiation dose of the physician and other staff who have to stay near the patient during the procedure. In our department, one of the senior interventionists had observed the hair loss in his lower limb. Thus the objective of this study was measuring the radiation dose of the physician's leg during common procedures in order to find out that whether the radiation dose exceeds the threshold of deterministic effects of radiation or not?.

Methods: Thermo luminescent-dosimeters (TLD-100) set on the leg part of the anthropomorphic whole body phantom PBU-50, was used in order to measure the radiation dose in 2 centers where our interventionist work. Meanwhile, the duration of exposure in important procedures was observed and recorded in center 1 for 2 months. During this period some data such as age, height, and weight of the patients and radiation exposure time, dose ( $\mathrm{mGy}$ ) and dose area product (dap) (Gy.cm²) was recorded

Results: The result of TLD dosimetry shown that the mean effective dose in center 1 and 2 was 0.20 and $0.86 \mathrm{mSv}$, and also the meandose $(\mathrm{mSv}) / \mathrm{min}$ in these centers was $0.02 \pm 0.00$ and $0.20 \pm 0.015 \mathrm{mSv} / \mathrm{min}$ respectively.

Conclusions: The effective annual dose of interventionists who work in center 1 and 2 was 143.08 and $1226.78 \mathrm{mSv}$ respectively. The high radiation dose which delivers to the leg of physicians in center 2 can be an important cause of radiation dermatitis occurred on the leg of our interventionist. Patient dose during most of the procedures was less than the threshold dose for occurring erythema but unfortunately in TIPS procedure which is a time-consuming procedure the patient radiation dose exceeds the threshold. It would be useful if a plan design to reduce the dose of patients and even physicians.
\end{abstract}

This is an abstract presented in the 33rd Iranian congress of radiology (ICR) and the 15th congress of Iranian radiographic science association (IRSA). 